\title{
Machine learning algorithms for distributed operations in internet of things IoT
}

\author{
Qusay Abdullah Abed ${ }^{1}$, Mohammed Thajeel Abdullah², Huda Jalil Dikhil ${ }^{3}$ \\ 1,2,3 Karbala Technical Institute, Al-Furat Al-Awsat Technical University, \\ 56001, Karbala, Iraq
}

\begin{abstract}
Generally, the things that have the great role in facilitating the emergence of internet-connected sensory devices can be embodied in the developments that happen in the sphere of software, hardware, and communication technologies. The internet-connected sensory devices present perceptions and measurements of data from the real world. It is suggested that nearly through 2020, the total use of internet-connected devices may reach to 25 to 50 billion. Actually, the relation between technologies and the volume of data being published is kept in one line. That is, if there is growth in the technologies, the volume of the data will be increased. Such technology, i.e. internetconnected devices, can be called as Internet of Things (IoT). Its role is to connect the real world with the cyber one. Furthermore, generating great data with velocity as its main characteristic will help in increasing the volume of IoT. To develop smart IoT applications, one can use such intelligent processing and analyzing such big data. In this paper, we tend to study the impact of implementing machine learning (ML) algorithms and methods and their efficiency in the IoT domain. As well as explore how these algorithms help in founding efficient backbone solutions to analyze and estimate the huge amounts of data that are expected to arise in the coming few years due to the rapid growth on demands for IoT based applications.
\end{abstract}

\begin{tabular}{ll}
\hline Keywords: & $\begin{array}{l}\text { Machine Learning, Internet of Things (IoT), Distributed Operations, Learning } \\
\text { Algorithms. }\end{array}$ \\
\hline
\end{tabular}

Corresponding Author:

Qusay Adullah Abed,

Karbala Technical Institute,

Al-Furat Al-Awsat Technical University,

56001, Karbala, Iraq.

E-mail: inkr.ks@atu.edu.iq

\section{Introduction}

Nowadays, many technologies have the chance to change the world. The Internet of Things (IoT) is one of them. In other words, it changes the way we behave against the world. The complicated things like sensors and chips are established in the real things that are around us. Additionally, it helps in getting us comprehend how these things work. In brief, it means that everything in the physical world is connected to the internet which in turns affect the way people live [1].

Additionally, making great businesses is another reason for having the present importance of IoT. In other words, it help in exploiting billions in this type of technology. In the previous years, the 
PEN Vol. 7, No. 4, December 2019, pp.1638- 1648

connected devices to the internet were more than humans. Yet in the following years, it is expected that the number will be increased to reach to 50 billion [2].

Economically, if people know how to link the cyber or digital worlds to the real one, there will be great business that may reach to $\$ 11.1$ trillion in a year. As such, companies have to invest in Internet of Things in order to be hopeful in distributing costs, redesigning factory workflows and improving tracing materials. For example, both UPS and John Deere are already using the Internet of Things (IoT) enabled fleet tracking technologies to improve supply efficiency and cut operating costs [3].

\section{Related work}

To understand running machine learning algorithms through the handheld devices is searching via Kargupta et al. [4] during the early years. Actually, such work presents proof of the potential of edge analytics even when tablets and smartphones have not been explore.

Generally, distributing the methods of machine learning among smartphones and (IoT) is investigating in a good way at the age of pocket data mining. Evidently, at this time the edge devices can be consider as complementary to another technology which is cloud computing as a means to scale out the systems of machine learning. Additionally, the spread of the methods of machine learning gives a chance for searching in this area [5]. Training small networks is the other approach which has the aim of lessening latency. An example of this approach is using mobile nets models from certain apps. Like Google. Actually, the developments that occur in this regard may cause a wide spread of developments in models of learning at the edge to decrease latency.

In the current paper, the researcher has focused on the methods of machine learning and how it can profile its performance. The methods are chosen according to the accuracy profiling of machine learning algorithms over a huge number of data sets [6].

\section{Machine learning for distributed operations}

The technology of machine learning can be describe as a sub-area of intelligence that is artificial. While the expression, i.e. machine learning, indicates the ability of Information Technology (IT) systems. It is so just to get suitable solutions to problems. That is, Information Technology System is enable by Machine learning for the sake of recognizing the patterns or the types depending on algorithms. Thus, one can generate a type of knowledge that is artificial basing on experience [7].

Several attempts were made to define machine learning from different aspects. Arthur Samuel in 1959 [8] defined machine learning as "Field of study that gives computers the ability to learn without being explicitly programmed".

Having its basis in the sphere of computer science, yet there are many variable methods in this regard. Actually, examples are very important in computer science since most of the learning depends on them. Figure 1 shows some data processing framework which has within it segmentation, preprocessing and noise removal. Where, the sensor sends the signal and then processed. Denoising and the features of extraction follow next and lastly the division level that provides an assessment or a judgment when the process ends [9].

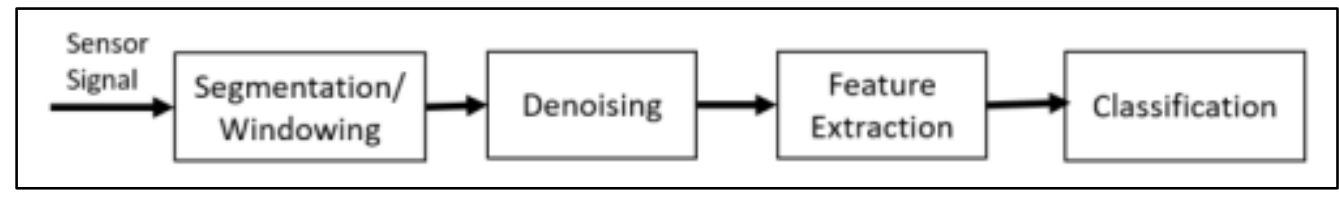

Figure 1. Basic signal processing framework including preprocessing, feature extraction and classification 
Naturally, mixed information is extracted through the stage of the feature extraction that bear bearing the data that is characterized by the parameters. While the machine learning trains the division stage to organize and divide the whole collection of features. In fact, such field like machine learning is wide and it has the chance to develop quickly and specially where it is emerged with other technologies like mobile devices [10]. Recently, the developments in this regard has attracted the attention of others in this area. There are several applications that succeed in keeping or having their own idiosyncrasies to have customize features. A large role has been performed by data compression and statistical signal. Further, this technology, i.e. machine learning, is divided into learning style and how data can be used [11].

- Supervised learning algorithms.

- Unsupervised learning algorithms.

This type of division is very significant in showing the input data role.

\section{Machine learning algorithms for internet of things (IoT)}

Generally, IoT [12] may be a framework of associated physical gadgets, shrewd machines or objects that have one of kind identifiers. The gadgets will ordinarily comprise of hardware, program, sensor, and radio empowering those things to persistently gather and exchange information. Radars or what the researcher has called them "sensors", which that comprise of a transducer that will change over a few shape of physical prepare into an electrical flag. Cases incorporate receivers, accelerometers, pressure sensors, cameras and thermometers etc.. Maybe a portable phone could be a great illustration of an associated gadget that implants a few heterogeneous sensors counting amplifier clusters, at slightest two cameras, magnetometers and accelerometers etc.. To begin with era shrewd phones for illustration ordinarily containing six sensors. Actually, a smart phone like Galaxy S5 may contain within it about 26 sensors. These sensors contain accelerometer, cameras, vicinity, magnetometer, IR, weight, stickiness, gyro etc. according to figure 2, that two sensors, magnetometer and Accelerometers are explored in several application, containing machine monitoring, the activity of human, the application of healthcare. Whereas, localization is being had by different parts of cooperative sensing and the learning machines.

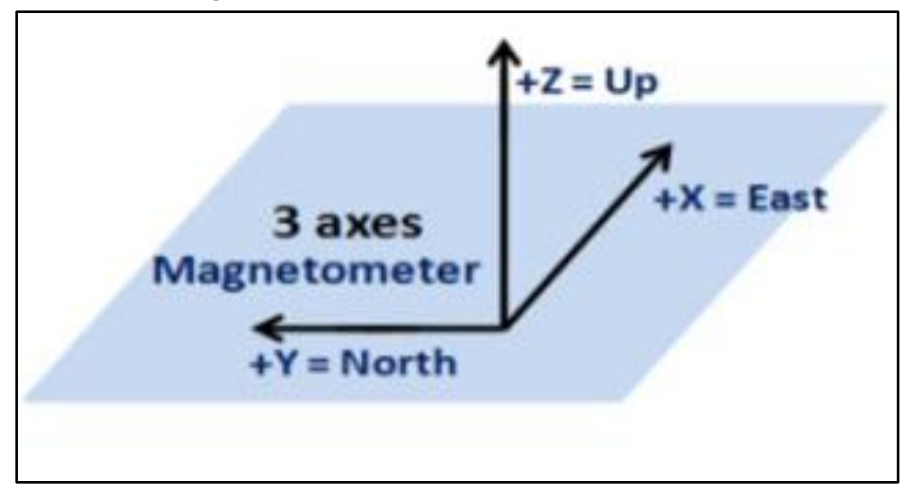

Figure 2. A magnetometer can help align with the earth's field

\subsection{Supervised learning}

There is another system that could be type of work through which input and desired yield data are presented. This system is calling Supervise learning. Input and yield data are name for classification to supply a learning evidence for information handling in the future. The expression "Supervised 
PEN Vol. 7, No. 4, December 2019, pp.1638- 1648 learnings" returns to the thought that an calculation is learning from a preparing dataset, which can be thought of as the teacher. The learning calculations are giving by supervised machine learning frameworks with known quantities to back future judgments. Self-driving cars, Chat bots, and other apps are within the frameworks that will use either supervised or unsupervised learning. Learning frameworks that are direct generally related with retrieval-based AI but they may too be competent of employing a generative learning show [13].

\section{How does supervised learning work?}

Generally, the way that supervised learning occurred is the input and output variables give a system the intentions of learning and how they are link to gether. The aim, here, is producing a correct linking of function when some new inputs are presented, then the output is predicted by the algorithm. Typically, it is a repeated operation, and each time the calculation makes an expectation, it is adjust or given input until it accomplishes an satisfactory level of execution.

A set of examples are include by training data with couples input and output that is desire. For instance, taking the application of image process in supervised learning, a system like AI could be supplied with variables pictures like those of cars or trucks. Having an enough amount of looking, there will be a chance to distinguish between false images where training is finished.

Actually, such apps can be divided into regression and classification. If the research has to give an example for classification, there will be just the above mentioned example. A regression problem is when the output is a true, computed value such as the price or weight. The most common supervised machine learning algorithms are discussed below.

\subsubsection{Linear regression}

If one comes to define linear regression, he can put the following definition: "it is a basic and commonly used type of predictive analysis". Two things are test in linear regression:

(1) Do a great work be done by through of a set of indicator factors in foreseeing a result (subordinate) variable?

(2) What type of factors can be pointers of the given results, and how they can perform their affect in the result variable?

Those regression estimates are using to show the relationship among one secondary variable and more than one free factors. The following formulae characterizes the relapse condition with one subordinate and one free variable: $\mathrm{y}=\mathrm{c}+\mathrm{b}^{*} \mathrm{x}$, where $\mathrm{y}=$ assessed subordinate variable score, $\mathrm{c}=$ steady, $\mathrm{b}=$ relapse coefficient and $\mathrm{x}=$ score on the free variable [14].

\subsubsection{Logistic regression}

Logistic regression can be defined as the appropriate regression test to conduct when the subordinate variable is dichotomous (parallel). Behaving similar to relapse investigations, the calculated relapse may be a prophetic investigation. In order to portray information, it is stated that calculated relapse can be utilized. Not only that, it can be used also to show the relation between one subordinate with twofold variable and more than one ostensible, ordinal, interim or ratio-level autonomous factors [15].

\subsubsection{Artificial neural networks (ANN)}

An artificial neural network (ANN) is an impersonation of the human brain. A normal brain has the capacity to memorize modern things, adjust to unused and changing environment. The brain has the 
PEN Vol. 7, No. 4, December 2019, pp.1638- 1648

foremost astounding capability to analyze fragmented and vague, fluffy data, and make its own judgment out of it. For case, we are able studied other's penmanship in spite of the fact that the way they compose may be totally distinctive from the way we type in. A child can distinguish that the shape of a ball and orange are both a circle. Indeed many days ancient child has the ability to recognize its mother from the touch, voice and scent. Able to identify a known individual indeed from a blurry photo. Brain may be an exceedingly complex organ that controls the complete body. The brain of indeed the foremost primitive creature has more capability than the foremost progressed computer. Its work isn't fair controlling the physical parts of the body, but too of more complex exercises like considering, visualizing, envisioning, envisioning, learning etc., exercises that cannot be put in real terms [16].

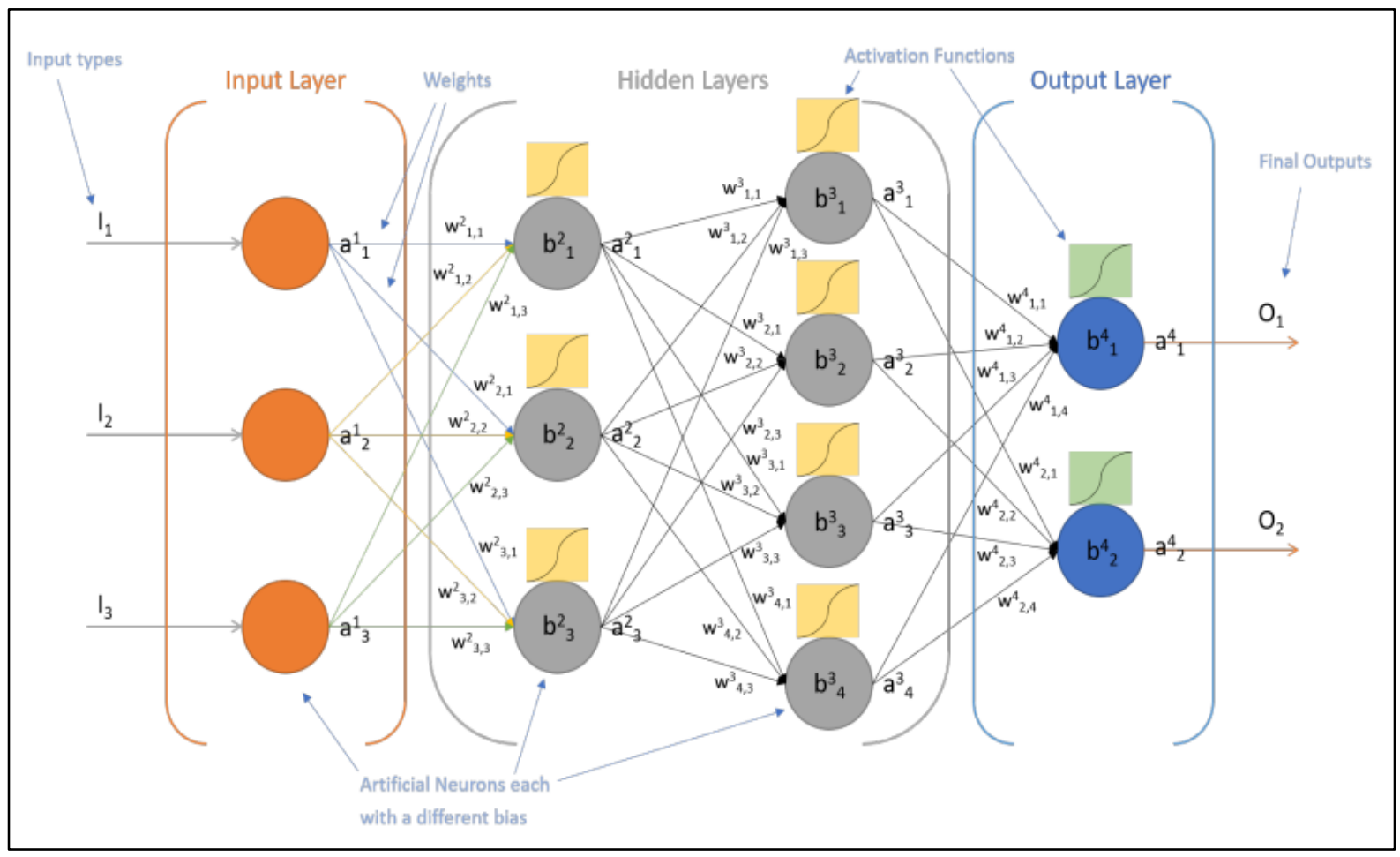

Figure 3. Visual representation of an artificial neural network

\subsubsection{Linear discriminant analysis}

This type of analysis, i.e. linear discriminant, can be described a dimensionality decrease strategy utilized as a preprocessing stage in the Machine Learning and the applications of design classification. The most objective of diminishment strategies is to diminish the measurements by evacuating the repetitive and subordinate highlights by changing the highlights from high to low dimensional spaces. Commonly, it can be used as dimensionality decreasing strategy within the step that can be classified as pre-processed for apps of machine learning and pattern-classification. The objective way is to extend a set of data into a space of low dimension category with great lesson distinctness in arrange dodge over-fitting ("curse of dimensionality") conjointly decrease computational charges [17].

\subsubsection{Decision tree}

A decision tree or what is called "a choice tree" could be a select bolster apparatus that employments a tree-like chart or demonstrate of a set of choices with their logical results, containing results of chance occasion, advantage costs, and value. It can be show as a way to reflect a calculation with its contents of conditional control articulations. Moreover, it can be indicated as a map for structure by 
which each axis indicates a test. Each department indicates a test result, etc. The ways from root to leaf speak to classification rule [18].

Tree based is considered as one of the best methods in this regard that is based on learning algorithms. Tree based strategies enable prescient models with tall precision, solidness and ease of elucidation. Not at all like direct samples, non-linear connections are outlined in a good way. They are versatile at tackling a type of issue at hand (classification or relapse) [19].

\subsubsection{Similarity learning}

When depicting pictures, people regularly resort to likeness characterizing the characteristics of the picture in relative terms instead of outright terms [20]. Unobtrusive contrasts between pictures can be demonstrating by a human effectively whereas totally describing a single picture could be a challenging assignment. Cognitive prove too recommends that we translate objects by relating them to prototypical illustrations put away in our brain. So likeness could be a crucial property and of incredible significance in recovery and categorization errands alike. Likeness learning is the method of deciding a work $d(a, b)$, which finds the ideal connection between two distinctive information things $a$ and $b$ in a quantitative way. In general, defining similitude for organized typical or numeric information is rather direct, but characterizing similitude from a human viewpoint is troublesome [21]. Typically particularly genuine for visual information for which there is a semantic hole between what a framework can extricate from the tangible information and the interpretations as the same data as for observers [22]. In this regard, likeness learning is seen as deciding a work $d(a, b)$ best coordinating the client translation of the information things and their relations [23].

\subsubsection{Bayesian logic}

According to Thomas Bayes, an English minister and mathematician, Bayesian logic could be a department of rationale connected to choice making and inferential insights that bargains with likelihood deduction: utilizing the information of earlier occasions to anticipate future occasions. Bayes to begin with proposed his hypothesis in his 1763 work (distributed two a long time after his passing in 1761), An paper towards fathoming an Issue within the convention of chances. Bayes' theorem given, for the primary time, a scientific strategy that might be utilized to calculate, given events in earlier trials, the probability of a target event in future trials. Concurring to Bayesian rationale, the as it were way to measure a circumstance with an questionable result is through deciding its likelihood [24].

\subsubsection{Support vector machines (SVM)}

Finding a hyperplane in $\mathrm{N}$-dimensional, ( $\mathrm{N}$ - the number of highlights) that unmistakably classifies the information focuses, space is the objective of the back vector machine calculation, To partitioned the two classes of information focuses, there are numerous conceivable hyperplanes that may be chosen. Our objective is to discover a plane that has the greatest edge, i.e. the greatest remove between information focuses of both classes. Maximizing the edge remove gives a few fortification so that future information focuses can be classified with more certainty. 


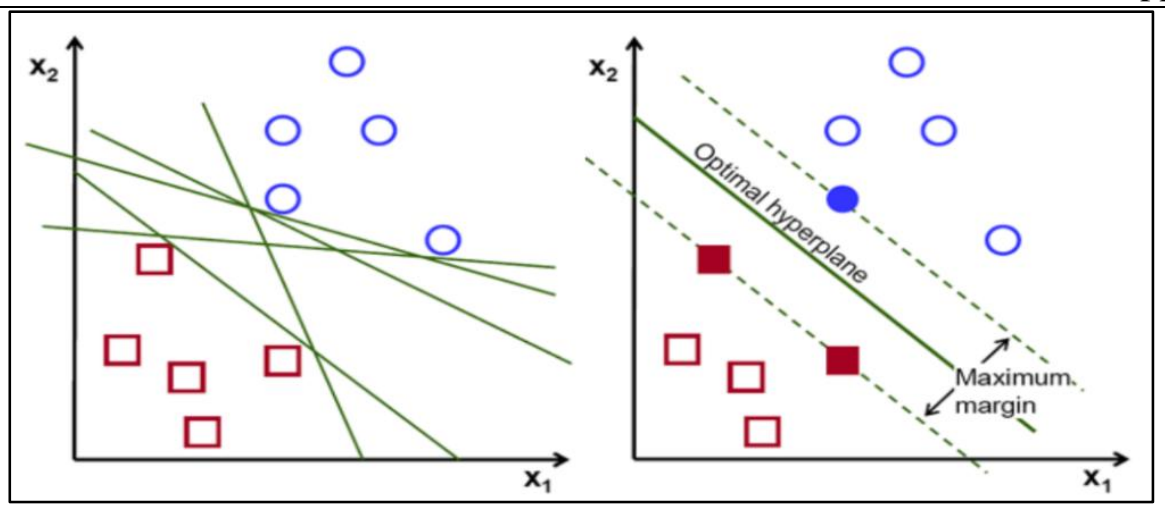

Figure 4. Possible hyperplane

\subsubsection{Random forests}

Having a relation to its name, random forest comprises of a huge quantity of person choice trees that work as an outfit. Each personal tree within the irregular timberland dribbles out a lesson forecast and the lesson with the foremost votes gets to be our sample's expectation as appeared within the following figure.

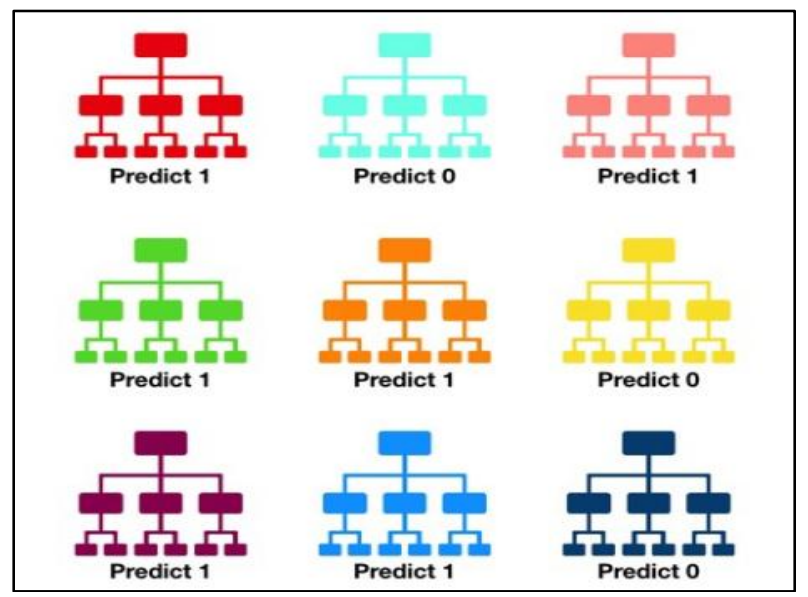

Figure 5. Visualization of a random forest model making a prediction

The main expression of random forest and what it has behind it may be a straightforward effective. In information science talk, the basic reason that the irregular timberland demonstrate works so well is:

"A large number of relatively uncorrelated models (trees) operating as a committee will outperform any of the individual constituent models."

Generally, it is said that key represents the low relation between models. Further, how can conjectures that are correlated like (stocks and bonds) gather to make selections that are worthy than of its parts. Sometimes, unrelated models or samples can present some expectations that are so precise. . The main cause for this excellent effect is that tree can protect each other (as long as they don't continually all blunder within the same heading). Whereas a few trees may be off base, numerous other trees are to be right, so as a bunch the trees are able to move within the redress course. So the prerequisites for irregular woodland to work well are:

1. A few genuine flag there must be in our highlights so samples built using these highlights do superior than random gambling.

2. The expectations that are doing by trees that are individual must have low relationships with each other. 
PEN Vol. 7, No. 4, December 2019, pp.1638- 1648 Choosing an algorithm as supervised learning requires many things. In other words, When choosing a administered learning calculation. There are a number of things that ought to be considered. The primary is the inclination and fluctuation that exists inside the calculation as there's a fine line between being adaptable sufficient and as well flexible [25]. Another is the complexity of the demonstrate or work that the framework is attempting to learn. Furthermore, the heterogeneity, exactness, repetition and linearity of the information ought to be analyzed some time recently choosing an calculation.

\subsection{Un-supervised Learning}

Talking about unsupervised algorithms [26], there are not unequivocal names related with the preparing a set of data. Actually, one can indicate that objective means drawing initiations from the internal information and after that demonstrate the fundamental structure and the dissemination within the information, in arrange to memorize more around the information. The most known illustration of an unsupervised calculation is clustering. The points of interest of the same is expressed as follow.

\subsubsection{Clustering}

Clustering [27] concerns itself within marking a design that can be suitable to dataset. In some cases, the strategy of clustering calculation bunches the given information into the number $\mathrm{K}$ of groups like the information focuses inside each group are comparative and information focuses from distinctive groups are disparate. Comparable to k-NN calculation, users may utilize of a similitude metric or remove metric. The letter $\mathrm{K}$ here indicates calculation is the best grouping calculations and is an instinctive and iterative calculation. It groups the information by isolating them into $\mathrm{K}$ bunches of rise to changes, minimizing the inactivity or within-cluster sum-of-squares. In any case, the calculation requires the number of clusters to be indicate some time recently running the calculation. Each perception or the information point is give to the cluster with the most near means that is called the Centroid of that cluster.

\subsubsection{Vector quantization}

Focusing on its less complex frame vector, quantization organizes information in courses and speaks about them through their centroids. It regularly employments a K-means grouping calculation to prepare the process of mapping. The centroids frame code words in addition to other code words are put away in a specific dictionary. "It may be a lossy compression strategy and is utilized in a few coding applications. As a result, the compressed information has blunders that are contrarily corresponding to thickness. This property is appeared in Figure 6 and compared with uniform quantization." Figure 7.

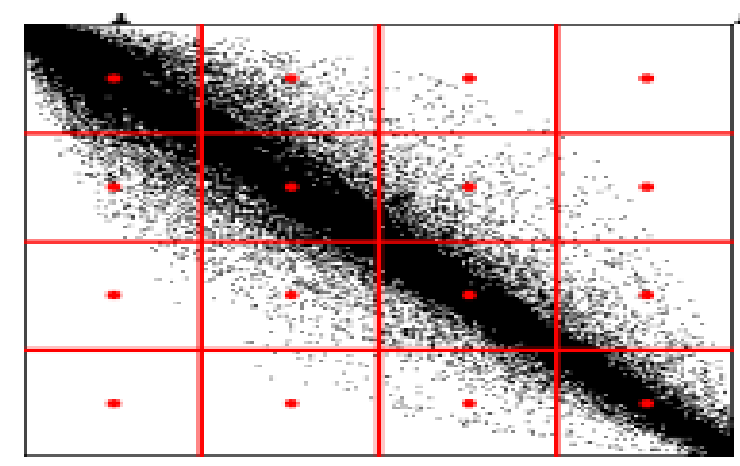

Figure 6. Uniform quantization of 2-dimensional data 


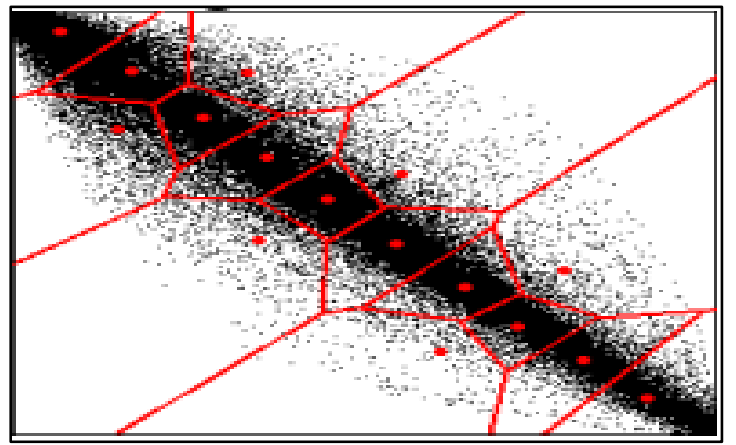

Figure 7. Vector quantization of 2-dimensional data

This procedure is utilizing in different discourse apps counting discourse coding, feeling acknowledgment, sound compression, large-scale picture classification and picture compression.

\subsubsection{Deep learning}

ANN, i.e. artificial neural networks, are broadly utilized within the regions of picture division, design acknowledgment and they have proved to be the foremost fruitful and they accomplish prevalent comes about in different areas counting flag preparing, computer vision, speech processing and normal dialect preparing. Profound learning may be a department in machine learning that has picked up notoriety very as of late, competent of learning different levels of reflection [28].

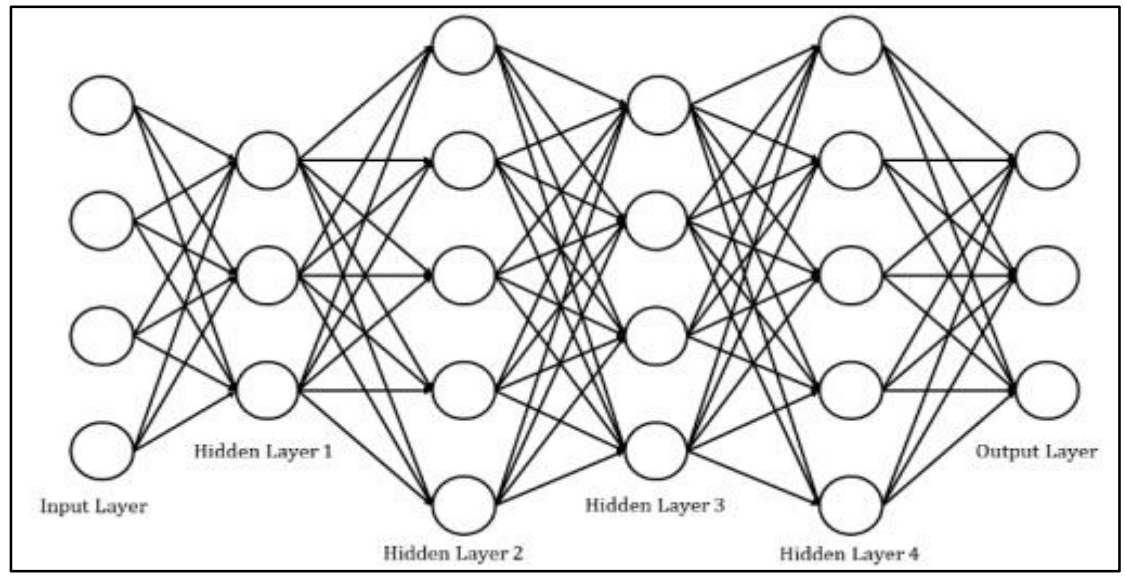

Figure 8. Artificial neural network with four hidden layers

In spite of the fact that, the beginning of neural network returns to in 1960, profound teaching picked up more notoriety since 2012 since of the extraordinary progressions within the strategy of GPUs and accessibility of huge branded sets of data. According to Figure 8, a basic manufactured nervous organize with four covered up strata are appeared. The final stratum, specifically the yield stratum, does the division. The terminology "deep learning" indicates many strata used to memorize numerous stages of illustration; each progressive stratum receipts the yield of the past stratum and bolsters the result to the following stratum [12].

\section{Conclusion}

IoT comprises of a tremendous number of diverse gadgets that are associated with each other and transmit gigantic sums of information through the enormous utilize of gigantic apps of IoT, and gives different administrations in spaces like vitality, versatility, and urban arranging. Those administrations may be optimize by analyzing the tremendous sums of information collected from these ranges. To 
PEN Vol. 7, No. 4, December 2019, pp.1638- 1648 extricate information from collected information, numerous machine-learning calculations can be connecte. Choosing an appropriate calculation for particular IoT or IoT app is an vital subject. In the current paper, numerous IoT machine learning ponders are checke on to call the current subject. About 3 truths ought to be taken in concern when applying ML calculations to keen information. The primary is that diverse apps in IoT and keen gadgets had their claim features, like the number of gadgets and sorts of the information that they produce. The moment is that the created information may present particular highlights that ought to be taken in concern. While the last is that the scientific categorization.

\section{References}

[1] M. H. Sharif, I. Despot, and S. Uyaver, "A proof of concept for home automation system with implementation of the internet of things standards," Periodicals of Engineering and Natural Sciences, vol. 6, pp. 95-106, 2018.

[2] M. H. Miraz, M. Ali, P. S. Excell, and R. Picking, "A review on Internet of Things (IoT), Internet of everything (IoE) and Internet of nano things (IoNT)," in 2015 Internet Technologies and Applications (ITA), 2015, pp. 219-224.

[3] S. Jagtap and S. Rahimifard, "Unlocking the Potential of the Internet of Things to Improve Resource Efficiency in Food Supply Chains," in International Conference on Information and Communication Technologies in Agriculture, Food \& Environment, 2017, pp. 287-301.

[4] H. Kargupta, B.-H. Park, S. Pittie, L. Liu, D. Kushraj, and K. Sarkar, "MobiMine: Monitoring the stock market from a PDA," ACM SIGKDD Explorations Newsletter, vol. 3, pp. 37-46, 2002.

[5] H. H. Nasereddin and M. FAQIR, "The impact of internet of things on customer service: A preliminary study," Periodicals of Engineering and Natural Sciences, vol. 7, pp. 148-155, 2019.

[6] M. Fernández-Delgado, E. Cernadas, S. Barro, and D. Amorim, "Do we need hundreds of classifiers to solve real world classification problems?," The Journal of Machine Learning Research, vol. 15, pp. 3133-3181, 2014.

[7] T. G. Dietterich, "Machine learning in ecosystem informatics and sustainability," in TwentyFirst International Joint Conference on Artificial Intelligence, 2009.

[8] P. L. Silsbee, A. C. Bovik, and D. Chen, "Visual pattern image sequence coding," IEEE Transactions on Circuits and Systems for Video Technology, vol. 3, pp. 291-301, 1993.

[9] U. S. Shanthamallu, A. Spanias, C. Tepedelenlioglu, and M. Stanley, "A brief survey of machine learning methods and their sensor and IoT applications," in 2017 8th International Conference on Information, Intelligence, Systems \& Applications (IISA), 2017, pp. 1-8.

[10] E. G. Ularu, F. C. Puican, G. Suciu, A. Vulpe, and G. Todoran, "Mobile Computing and Cloud maturity-Introducing Machine Learning for ERP Configuration Automation," Informatica Economica, vol. 17, 2013.

[11] J. L. Berral-García, "A quick view on current techniques and machine learning algorithms for big data analytics," in 2016 18th international conference on transparent optical networks (ICTON), 2016, pp. 1-4.

[12] I. Muhic and M. Hodzic, "Internet of Things: Current Technological Review," Periodicals of Engineering and Natural Sciences, vol. 2, 2014.

[13] M. Rouse, "Supervised learning," ed, 2019.

[14] P. Sedgwick, "Simple linear regression," BMJ, vol. 346, 2013.

[15] S. Sperandei, "Understanding logistic regression analysis," Biochemia medica: Biochemia medica, vol. 24, pp. 12-18, 2014. 
[16] H. P. Singh, C. A. Bailer-Jones, and R. Gupta, "Introduction to artificial neural networks," 2001.

[17] L. Wu, C. Shen, and A. Van Den Hengel, "Deep linear discriminant analysis on fisher networks: A hybrid architecture for person re-identification," Pattern Recognition, vol. 65, pp. 238-250, 2017.

[18] "Decision Trees-A simple way to visualize a decision," 02-Oct-2019 2019.

[19] N. V. Chawla, "Data mining for imbalanced datasets: An overview," in Data mining and knowledge discovery handbook, ed: Springer, 2009, pp. 875-886.

[20] J. P. Gee, "Learning and games," The ecology of games: Connecting youth, games, and learning, vol. 3, pp. 21-40, 2008.

[21] Y. Yang, S. Liao, Z. Lei, and S. Z. Li, "Large scale similarity learning using similar pairs for person verification," in Thirtieth AAAI Conference on Artificial Intelligence, 2016.

[22] G. P. Nguyen, M. Worring, and A. W. Smeulders, "Interactive search by direct manipulation of dissimilarity space," IEEE Transactions on Multimedia, vol. 9, pp. 1404-1415, 2007.

[23] E. J. Sommerfeldt, "The dynamics of activist power relationships: A structurationist exploration of the segmentation of activist publics," International Journal of Strategic Communication, vol. 6, pp. 269-286, 2012.

[24] "What is Bayesian logic? - Definition from WhatIs.com." [Online]. Available: https://whatis.techtarget.com/definition/Bayesian-logic., [Accessed: 04-Oct-2019].

[25] Y. OUASSIT, S. ARDCHIR, and M. AZOUAZI, "How Machine Learning Potentials are transforming the Practice of Digital Marketing: State of the Art," Periodicals of Engineering and Natural Sciences, vol. 6, pp. 373-379, 2018.

[26] M. Khanum, T. Mahboob, W. Imtiaz, H. A. Ghafoor, and R. Sehar, "A survey on unsupervised machine learning algorithms for automation, classification and maintenance," International Journal of Computer Applications, vol. 119, 2015.

[27] S. Logeswari, K. Premalatha, and D. Sasikala, "A Survey on Text Mining in Clustering," International Journal of Advanced Research in Computer Science, vol. 2, 2011.

[28] I. Goodfellow, Y. Bengio, and A. Courville, Deep learning: MIT press, 2016. 